\title{
Numerical analysis of frazil ice formation in turbulent convection
}

\author{
A. Abbà ${ }^{1}$, M. Montini ${ }^{1}$, L. Pignagnoli ${ }^{2,3}$, L. Valdettaro $^{1}$, and P. Olla ${ }^{4}$ \\ 1 Dipartimento di Matematica, Politecnico di Milano, Piazza L. da Vinci 32, I-20133 Milano, Italy \\ 2 ISAC-CNR, I-40129 Bologna, Italy \\ ${ }^{3}$ Dipartimento di Matematica, Università degli Studi di Milano, I-20133 Milano, Italy \\ ${ }^{4}$ ISAC-CNR and INFN, Sez. Cagliari, I-09042 Monserrato, Italy
}

\section{Abstract}

We study the first steps of ice formation in fresh water turbulent convection (frazil ice regime). We explore the sensitivity of the ice formation process to the set of non-dimensional parameters governing the system. We model the mixture of ice crystals and water as a two-phase medium composed of water and ice particles of fixed diameter. We use the Boussinesq approximation and we integrate numerically the set of equations making use of a numerical code based on second order finite difference. A dynamic LES model for the subgrid scales is used.

We show that the ice particle rise velocity and the ice concentration source term coefficient significantly affect the frazil ice dynamics. The maximum of ice production is obtained in those situations where the rise velocity is of the same order of magnitude of a characteristic velocity of the thermal downwelling plumes. We develop a simple model which captures the trend of the growth rate as a function of the relevant parameters. Finally we explore the nonlinear regime and we show that the parameter which plays a key role in fixing the concentration value at the statistically steady state is the heat exchange source term coefficient.

\section{Introduction}

The first stage of ice formation at the supercooled free surface of oceans, rivers and lakes is called frazil ice, a suspension of individual, small randomly-oriented crystals typically measuring approximatively $1 \div 4 \mathrm{~mm}$ in diameter and $1 \div 100 \mu \mathrm{m}$ in thickness [10, 13].

Frazil ice evolves rapidly into a thin slurry of ice platelets giving the sea a greasy or milky appearance, known as grease ice. As cooling continues and the density of the frazil suspension increases, the frazil crystals freeze together into small cakes. Collisions between neighbouring cakes forces frazil ice over the cake edges where rims form to give the characteristic form of pancake, hence the appellation pancake ice. Initially formed pancakes may be only a few centimeters in diameter, but they grow in diameter and thickness from surrounding frazil matrix and may reach $3 \div 5 \mathrm{~m}$ in diameter and $50 \div 70 \mathrm{~cm}$ in thickness. The last stage of sea ice evolution is the coalescence of pancake ice floes, which aggregate into a continuous ice sheet. In figure (11) a picture of a frazil-pancake ice field in Polar ocean is reported.

In rivers, frazil ice production is a transient phenomena which occurs in turbulent supercooled water. The first stage is the water temperature lowering to about $0.01 \div 0.1^{\circ} \mathrm{C}$ below the freezing point because of the wind action or the radiative cooling; then the frazil discs start to form and the water temperature following the mass of crystals increases in the order of minutes toward the freezing point. The presence of frazil ice can cause serious problems to hydroelectric facilities such as the blocking of turbine intakes, the blockage of hydroelectric reservoirs and the freezing open of gates.

In polar ocean, frazil ice can form under ice covers and in open region within polar pack ice, more precisely in 100 meters scale leads and in kilometer scale polynyas. Frazil ice is typically present in the Marginal Ice Zone (MIZ), which is the transition region between the open polar ocean and the continuous ice that covers the central basin. Due to the combined action of wind 
and surface waves, the turbulent waters of MIZ prevent the formation of large ice sheets. The presence of frazil ice fields is associated with important geophysical and biological processes of the polar oceans so that the study of frazil ice dynamics is an active research field. As frazil ice production is accompanied by salt rejection, it is believed that it could play an important role in stimulating the convection process of ocean waters. Moreover, frazil ice plays an important role as scouring and sediment transfer agent and also it can transport biological species from the sea bottom to the surface pack ice.

This paper is organized as follows. Section 3 is devoted to a brief description of the frazil ice formation process. In section 4 the derivation of the frazil ice transport model is reported, while section [5] is dedicated to the Boussinesq-like approximation. In section [6] we present numerical results of the simulations and finally section 7 is devoted to the conclusions.

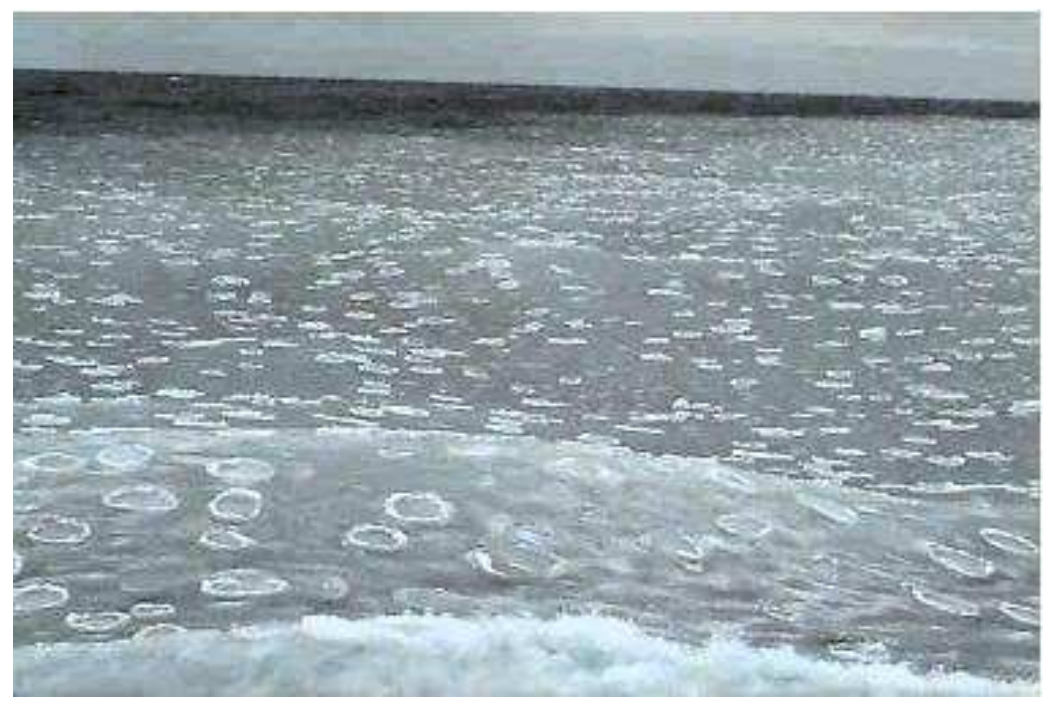

Figure 1: Frazil-pancake ice field in Polar ocean (www.vims.edu/bio/microbial/NBPice.html).

\section{The formation of frazil ice}

The time it takes for ice formation in oceans, rivers and lakes can be quite different from one year to the other, due to meteorological variations. Estimating this time is a very complicated task, which involves many parameter such as wind, water temperature and water depth. Immediate applications of this problem can be found in shipping and ice breaking service. To this end, many theoretical and experimental studies were performed during the last decades, giving useful information about time histories of temperature and ice formation.

Designers of hydraulics structures such as canals, water transport facilities and hydroelectric power structures are particularly interested in three ice-flow regimes, which are common in rivers during freeze-up period: continuous ice sheet, floating frazil ice and well mixed flow (with frazil ice crystals vertically mixed in the water flow). In the last case, if the velocity of the river is sufficiently large, open water conditions may persist throughout the winter. In consequence of this, a very important task is to predict, starting from a given set of flow conditions, which kind of regime could be expected, and the transition from an ice cover to open water. Obviously, when frazil ice crystals remain well mixed with the fluid, they cannot accumulate beneath the surface and the ice cover can not form, so the conditions under which the transition from well mixed flow to floating ice layer occurs, could be employed as a rough criteria for the formation of the continuous ice cover. In [5] the rough criteria cited above was compared, with good agreement, with experimental and field data. In particular, it has been remarked that the presence or absence 
of the well mixed regime could be forecasted comparing the buoyancy time scale $T_{B}$ with the vertical turbulent diffusive mixing scale $T_{D}$. When $T_{B}<T_{D}$ a layered flow will develop, because the frazil ice crystals rise to the water surface faster than they are removed by turbulent mixing. Under the same argument, the opposite case $T_{B}>T_{D}$ corresponds to a well mixed flow. More sophisticated criteria take into account other hydrological aspects, such as sinuosity, channel and slope variability, the transition from frazil ice layer to ice cover throughout pancake ice formation and the mechanics of interaction between the different kinds if ice [5].

In [15] a numerical simulation of ice formation and transport in turbulent ocean water was performed. The upper ocean was modelled as an Ekman layer, using a $k-\epsilon$ turbulence model for the Reynolds-averaged Navier-Stokes (RANS) equations. This model was improved in [19] considering the interaction between frazil ice particles of different size. In [9] a model of frazil ice transport under an ice shelf was proposed and a Boussinesq-like approximation was adopted in the limiting case of very small ice concentration. In this model the vertical structure was simplified by assuming all properties to be constant over the thickness of the plume and discontinuous at its boundaries; the problem was reduced to one dimension. In [18 the model was extended to the case of ice particles of different size. More recently [17, a Large Eddy Simulation (LES) with a Smagorinsky turbulence model was carried out in order to study frazil ice dynamics and the ice effects on turbulent structures under polar ice covers and in leads. This work took into account the effects of salinity. Finally [6] presented results of LES simulations for a turbulent flow with frazil ice crystals of different size.

In our work we propose a parametric study of the problem in the framework of Boussinesq approximation. In the case of large ice concentrations, the particles interaction at the ice crystal microscale should be considered. In particular, it was observed that this phenomenon becomes relevant for ice volume concentrations above .003 [15]. Moreover, the presence of frazil ice crystals produces an increment in the ice-water mixture effective viscosity. In [3] it was shown that, for small ice concentrations, this parameter differs from that of pure fluid by a value of the same order of the concentration itself.

In this paper we have used a convection model for frazil ice transport in turbulent water by considering the mixture of ice crystals and water as a two-phase medium. The balance equations for mass, momentum and energy of the ice-water mixture are supplemented by an equation for the temporal evolution of the ice mass concentration. This equation is strongly coupled to the others. In particular, the ice particles melting/freezing process and the temperature fluctuations modify the density of the mixture and thus the buoyancy force, which, in turn, affects the hydrodynamical behavior.

The simulation of these equations is subject to numerical instability due to large round-off errors. In order to avoid this behavior, related to the large magnitude of the coefficient of the buoyancy term, we have applied a Boussinesq-like approximation to the equations of motion.

A complete model of frazil ice dynamics should be able to represent the complex processes involved in the ice formation, like growth of ice crystals, flocculation (the clustering of two, or more, frazil particles), break-up (the cracking of an ice particle) and secondary nucleation (the production of small ice crystals by the ice fragments scavenging from crystal surface). As a consequence, a large range of particles size distribution is expected with the crystal diameters varying from micrometer to millimeter. In this paper, however, we have considered only one particle size. This is not a crude simplification, because the small ice crystals rapidly evolve into larger ones and finally only ice particles within a narrow size range actively affect the system dynamics [9].

For the sake of simplicity we limit our analysis to ice formation in fresh water and we postpone studying the effect of salinity to a future work.

We have integrated numerically the evolution equations in the Boussinesq limit using a second order finite difference method. The smallest scales have been modelled within the Large Eddy Simulation approach: the equations for the resolved scales are obtained introducing a spatial filter that removes the unresolved small scales of turbulence; the filtered nonlinear terms are modelled using the approach of 20. Numerical simulations have been carried on to determine the sensitivity of ice growth rate and saturation process to different sets of non-dimensional parameters. 


\section{Model equations for frazil ice transport in turbulent wa- ter}

We model the convective motion in the upper ocean mixed-layer as convection between two parallel and infinitely long boundaries (albeit periodic), with the upper boundary corresponding to the air-ocean interface and the lower one corresponding to the mixed layer bottom. The distance $L$ between the boundaries and the temperature difference $\Delta T$ maintained between them are set equal, respectively, to a typical depth of the mixed-layer $(30 \div 100 \mathrm{~m}$. $)$ and to a typical temperature gap between the air-ocean interface and the mixed-layer bottom $\left(0.1 \div 0.2^{\circ} \mathrm{C}\right)$. The temperature at the air-ocean interface is set to a value slightly less than the salt water freezing point $-2.0^{\circ} \mathrm{C}$ corresponding to a typical salinity value $S_{0}=34.5 \mathrm{psu}$. Salinity variations play only a small role in the thermodynamics of the system and we shall hold it constant 6]. Ice concentration is assumed to be large enough to be represented as a continuum but small enough to neglect the particles interactions. As a consequence, the system of water and frazil ice particles can be modelled as a two-component mixture, which is treated as a locally homogeneous fluid with averaged properties. In the dilute limit that we are considering, the effective viscosity of the ice-water mixture differs from that of pure fluid by a value of the same order of the concentration itself [3] and this viscosity increment can be neglected.

We consider a frame of reference such that the $z$ axis is vertically upward. The ice-water mixture density $\rho$ is given by:

$$
\rho=\rho_{i} \phi_{i}+\rho_{w} \phi_{w}
$$

where $\rho_{i}$ and $\phi_{i}$ are, respectively, the ice density and volume fraction; similarly $\rho_{w}$ and $\phi_{w}=1-\phi_{i}$ are, respectively, the water density and volume fraction; $\rho_{w}$ is related to the temperature by the following equation of state:

$$
\rho_{w}=\rho_{w 0}\left[1-\alpha\left(T-T_{0}\right)\right]
$$

where $\rho_{w 0}$ is the density corresponding to the lower boundary temperature $T_{0}$ and $\alpha$ is the thermal expansion coefficient.

The total density $\rho$ can be written as:

$$
\rho=\left(\frac{C_{i}}{\rho_{i}}+\frac{1-C_{i}}{\rho_{w}}\right)^{-1}
$$

in terms of $C_{i}$, that is the ice mass concentration, obeying $C_{i} \rho=\rho_{i} \phi_{i}$ ( $\operatorname{similarly} C_{w}=1-C_{i}$ will denote the water mass concentration).

The equation of continuity for the total density $\rho$ is given by:

$$
\frac{\partial \rho}{\partial t}+\nabla \cdot(\rho \mathbf{u})=0
$$

while the equation for the mixture momentum balance is formulated as follows:

$$
\rho \frac{\partial \mathbf{u}}{\partial t}+\rho(\mathbf{u} \cdot \nabla) \mathbf{u}=-\nabla p+\rho g \vec{k}+\nu \rho \nabla^{2} \mathbf{u}
$$

where $\mathbf{u}$ and $p$ are respectively the velocity and the pressure fields, $g$ is the gravity acceleration and $\nu$ is the kinematic viscosity.

The equation for the temperature $T$ can be written as follows:

$$
\frac{\partial T}{\partial t}+\mathbf{u} \cdot \nabla T=k_{T} \nabla^{2} T+G_{T},
$$

where $k_{T}$ is the water thermal diffusivity and $G_{T}$ is the source term due to melting and freezing processes, considering the ice particle as a sphere: 


$$
G_{T}=\frac{\Delta T}{\Delta t}=\lambda_{T} \rho C_{i}\left(T_{i}-T\right)
$$

where [7:

$$
\lambda_{T}=\frac{3 \bar{h}}{R_{S} c_{p} \rho_{w} \rho_{i}}
$$

and $\bar{h}=\frac{N u k_{w}}{2 R_{S}}$ is the heat transfer coefficient $\left(N u\right.$ is the Nusselt number and $k_{w}$ is the sea water thermal conductivity, $R_{S}$ is the ice particle radius), $T_{i}$ is the ice particle temperature, taken equal to the freezing point, and $c_{p}$ is the water specific heat.

Finally, the equation for the ice component can be written as ([8], [11, [15]):

$$
\frac{\partial\left(\rho C_{i}\right)}{\partial t}+\nabla \cdot\left(\mathbf{u} \rho C_{i}\right)=w_{r} \frac{\partial\left(\rho C_{i}\right)}{\partial z}+k_{i} \nabla \cdot\left(\rho \nabla C_{i}\right)+G_{i},
$$

where $k_{i}$ is the ice molecular diffusivity, $\mathbf{w}_{\mathbf{r}}=\left(0,0, w_{r}\right)$ is the rise velocity that derive from the balance between the upward net buoyancy force and the drag force. It is still an open question how to model this velocity; we have left it as a free parameter for sensitivity analysis ([1], [8, [15, [19]). Here $G_{i}$ is the source term due to melting and freezing processes and it is given by:

$$
G_{i}=\frac{\Delta\left(\rho C_{i}\right)}{\Delta t}=\lambda_{i} \rho C_{i}\left(T_{i}-T\right)
$$

where 7:

$$
\lambda_{i}=\frac{3 \bar{h}}{R_{S} L_{i} \rho_{i}}
$$

and $L_{i}$ is the ice latent heat of fusion.

It is important to remark that we have used spherical particles instead of disk-like ones which better represent the frazil ice platelets. The choice is motivated by the great simplifications introduced in the model. Moreover the ice particle shape affects the source terms only in their magnitude; the latter parameters will be used in the foregoing for the sensitivity analysis and they will vary in a wide range; so we can conclude that the effect of the particle shape on the source terms is not significant for the analysis we are going to carry on.

In order to rewrite eqs. (29) in non-dimensional form, we consider the depth of the upper ocean mixed-layer $\bar{L}=L$ as length scale, so the depth of the layer is 1 , the viscous scale velocity $\bar{U}=\nu / \bar{L}$ as velocity scale, the corresponding time $\bar{t}=\bar{L} / \bar{U}=\bar{L}^{2} / \nu$ as time scale, the water density $\bar{\rho}=\rho_{w 0}$ as density scale and the temperature gap $\overline{\Delta T}$ between upper and lower boundary as temperature scale. We indicate by $z$ the dimensionless vertical coordinate with $z_{0}=-1 / 2$ the lower boundary. We obtain:

$$
\begin{gathered}
\rho_{w}=1-\bar{\alpha}\left(T-\widehat{T}_{0}\right), \\
\rho=\left(\frac{C_{i}}{\rho_{i}}+\frac{1-C_{i}}{\rho_{w}}\right)^{-1} \\
\frac{\partial \rho}{\partial t}+\nabla \cdot(\rho \mathbf{u})=0 \\
\rho \frac{\partial \mathbf{u}}{\partial t}+\rho(\mathbf{u} \cdot \nabla) \mathbf{u}=-\nabla p-\frac{R a}{\bar{\alpha} P r} \rho \vec{k}+\rho \nabla^{2} \mathbf{u}, \\
\frac{\partial T}{\partial t}+\mathbf{u} \cdot \nabla T=\frac{1}{P r} \nabla^{2} T+\beta \rho C_{i}\left(\widehat{T}_{i}-T\right), \\
\frac{\partial\left(\rho C_{i}\right)}{\partial t}+\nabla \cdot\left(\mathbf{u} \rho C_{i}\right)=\widehat{w}_{r} \frac{\partial\left(\rho C_{i}\right)}{\partial z}+\frac{1}{S c} \nabla \cdot\left(\rho \nabla C_{i}\right)+\gamma \rho C_{i}\left(\widehat{T}_{i}-T\right),
\end{gathered}
$$


where $R a=g \bar{\alpha} \bar{L}^{3} /(k \nu)$ is the Rayleigh number, $\operatorname{Pr}=\nu / k$ is the Prandtl number, $S c=\nu / k_{i}$ is the ice Schmidt number, $\widehat{w}_{r}=w_{r} / \bar{U}, \bar{\alpha}=\alpha \overline{\Delta T}, \beta=\frac{\lambda_{T} \bar{L}^{2} \bar{\rho}}{\nu}, \gamma=\frac{\lambda_{i} \overline{\Delta T} \bar{L}^{2}}{\nu}, \widehat{T}_{i}=T_{i} / \overline{\Delta T}$ and $\widehat{T}_{0}=T_{0} / \overline{\Delta T}$.

At the lateral boundaries, we prescribe periodic conditions, while at the horizontal boundaries we impose a fixed temperature and free-slip condition for velocity. For frazil ice concentration we impose Dirichlet conditions $C_{i}=0$ at the bottom boundary, while the conditions to impose at the top is still an open question; we postpone the discussion of such boundary conditions at section 6.1 and 6.4

\section{The Boussinesq approximation for frazil ice transport model}

The buoyancy term in the mean momentum balance equation (13) is multiplied by the factor:

$$
\frac{R a}{\bar{\alpha} P r} \text {. }
$$

In the cases of physical interest, the Rayleigh number is in excess of $10^{8} \div 10^{9}$ and $\bar{\alpha} \simeq 10^{-6}$; hence this factor can be estimated to have a magnitude of at least $10^{14} \div 10^{15}$. As a consequence, the numerical evaluation of this term in a double precision code is subject to significant round-off errors: this produces severe numerical instability. In order to avoid this problem we introduce a Boussinesq-like approximation. We introduce a hydrostatic equilibrium without ice and then we write down the equations for the small perturbations to this state. The fluctuations are written as a power series of the small parameter $\bar{\alpha}$ and the expansion is truncated to lowest order. Temperature fluctuations, pressure and velocity field are of order $\mathcal{O}(1)$, whereas density and ice concentration fluctuations are of order $\mathcal{O}(\bar{\alpha})$. We thus have:

$$
\begin{gathered}
\mathbf{u}=\mathbf{u}^{\prime}+\mathcal{O}(\bar{\alpha}), \quad \nabla p=-\frac{R a}{\bar{\alpha} P r}\left[1+\bar{\alpha}\left(z-z_{0}\right)\right] \vec{k}+\nabla p^{\prime}+\mathcal{O}(\bar{\alpha}), \\
T=\widehat{T}_{0}-\left(z-z_{0}\right)+T^{\prime}+\mathcal{O}(\bar{\alpha}), \quad C_{i}=\bar{\alpha} C_{i}^{\prime}+\mathcal{O}\left(\bar{\alpha}^{2}\right), \quad \rho=1+\bar{\alpha}\left(z-z_{0}\right)+\bar{\alpha} \rho^{\prime}+\mathcal{O}\left(\bar{\alpha}^{2}\right) .
\end{gathered}
$$

Substituting into eq. (11) to leading order in $\bar{\alpha}$ we obtain:

$$
\rho^{\prime}=-\left(\frac{1-\rho_{i}}{\rho_{i}} C_{i}^{\prime}+T^{\prime}\right)
$$

and the equations (12) and (13) become:

$$
\begin{gathered}
\nabla \cdot \mathbf{u}^{\prime}=0 \\
\frac{\partial \mathbf{u}^{\prime}}{\partial t}+\left(\mathbf{u}^{\prime} \cdot \nabla\right) \mathbf{u}^{\prime}=-\nabla p^{\prime}+\frac{\operatorname{Ra}}{\operatorname{Pr}}\left[\frac{1-\rho_{i}}{\rho_{i}} C_{i}^{\prime}+T^{\prime}\right] \vec{k}+\nabla^{2} \mathbf{u}^{\prime} .
\end{gathered}
$$

Note that the factor $\frac{R a}{\bar{\alpha} P r}$ in the buoyancy term of eq. (13) has been transformed to $\frac{R a}{P r}$, which is not subject anymore to numerical instability due to round-off errors.

Temperature equation (14) becomes:

$$
\frac{\partial T^{\prime}}{\partial t}+\mathbf{u}^{\prime} \cdot \nabla T^{\prime}=-w^{\prime}+\frac{1}{\operatorname{Pr}} \nabla^{2} T^{\prime}+\beta \bar{\alpha} C_{i}^{\prime} f\left(z, T^{\prime}\right)
$$

where:

$$
f\left(z, T^{\prime}\right)=\widehat{T}_{i}-\widehat{T}_{0}-\left(z-z_{0}\right)-T^{\prime}
$$

and finally the ice transport equation (15) reduces to: 


$$
\frac{\partial C_{i}^{\prime}}{\partial t}+\mathbf{u}^{\prime} \cdot \nabla C_{i}^{\prime}=\frac{1}{S c} \nabla^{2} C_{i}^{\prime}+\widehat{w}_{r} \frac{\partial C_{i}^{\prime}}{\partial z}+\gamma C_{i}^{\prime} f\left(z, T^{\prime}\right) .
$$

Equations (1720) are a complete set of equations for the small perturbations to the hydrostatic equilibrium. For sake of simpler notations, we shall drop from now on the primes from the variable names.

\section{$6 \quad$ Numerical results}

Equations (1720) were solved numerically using a fractional step approach [12] with a third order, three steps, explicit Runge-Kutta scheme. For the spatial discretization, we have employed a second order accurate finite difference scheme on a staggered grid. For the momentum equation, each Runge-Kutta time step has been splitted into two steps; the first one consists in the calculation of a temporary velocity field without making use of the pressure terms; the second step is a projection of the solution onto a divergence-free space, which results in solving a Poisson equation for pressure. A fast Poisson solver using Fourier and cycling reduction method was adopted [16].

The effects of the small unresolved scales on the resolved ones are modelled by using the Subgrid Scale Model proposed by [20]. We utilize the same subgrid scale model for the flux of ice concentration and for the temperature: the turbulent Schmidt number is computed by using the dynamic procedure and varies in space and time.

The domain has an extension of $2 \pi$ in the horizontal directions and height equal to one. The resolution varies with Rayleigh number: at $R a=10^{4}, 10^{6}$ and $10^{8}$ we used respectively $31 \times 31 \times 30$, $51 \times 51 \times 36$ and $81 \times 81 \times 60$ collocation points.

\subsection{Ice production in deep water}

A significant amount of oceanic frazil ice might be produced in the deep layers of water. In 13 , for example, it is stated that a large amount of frazil ice in seawater adjacent to shelves could be produced by freezing of parcels of water rising towards supercooled upper layers. In this series of simulations, we want to focus on the ice produced in the regions far from the upper layer. To this end we use the boundary conditions $C_{i}=0$ at $z= \pm 1 / 2$. These conditions amount to cancel the convective flux of ice through the horizontal boundaries. They produce a very thin boundary layer immediately beneath the free surface where a non-physical ice concentration drop is present.

In all the subsequent simulations we assume that the freezing temperature is $\widehat{T}_{i}=\widehat{T}_{0}+z_{0}$, that is the upper half of the hydrostatic layer is below freezing point.

Typical curves of the total ice concentration versus time are shown in Fig. 2 In the left figure, we clearly distinguish a short transient followed by an exponential growth, ended by the nonlinear saturation. Evolution of kinetic energy is shown in Fig. 3 The ice seeds are introduced at time $t=0$. We observe that the production of ice is fast compared to the large eddies turnover time. As a consequence, during the linear phase, the velocity and temperature fields are almost constant and we get an almost pure exponential growth of ice concentration. In the right figure, instead, the exponential growth phase does not appear very clearly. This is because the time scale for the ice formation is large compared to the convection turnover time: the flow changes significantly during ice formation. The fast and slow regimes appear very clearly in Fig. [3.

In the fast growth regime, we observe that most of the ice is produced inside the strongest

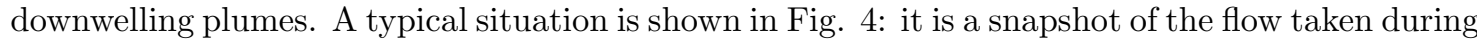
the linear phase of ice growth. The isovalue for temperature fluctuation has been chosen so that only the most intense downwelling plume appears (since the flow is periodic in horizontal directions the four large green structures are actually contiguous). We see that ice (white structures) is present mostly at the top of that plume. This is natural, since it is also the coldest place inside the flow: the largest part of ice is produced there, and, since the time scale for the linear phase is small, the shape of the plume almost does not change and the ice remains confined inside it. By contrast, in the slow regime, the spots of large ice concentration do not necessarily reside in 


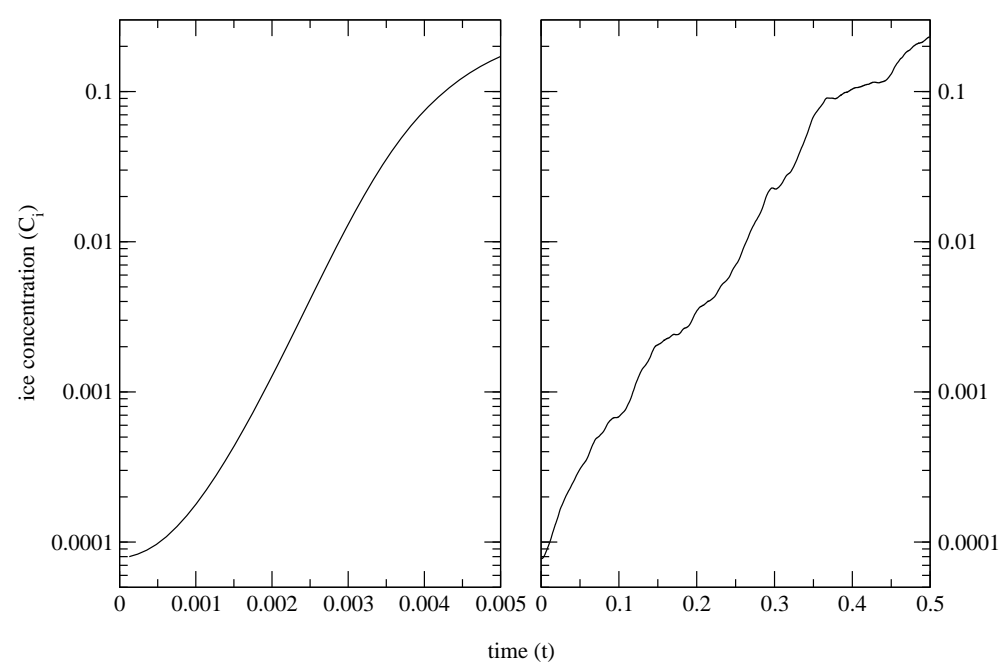

Figure 2: Evolution of total ice concentration for $R a=10^{6}$. Left: $\gamma=10^{4}, w_{r}=150$. Right: $\gamma=10^{3}, w_{r}=10$.

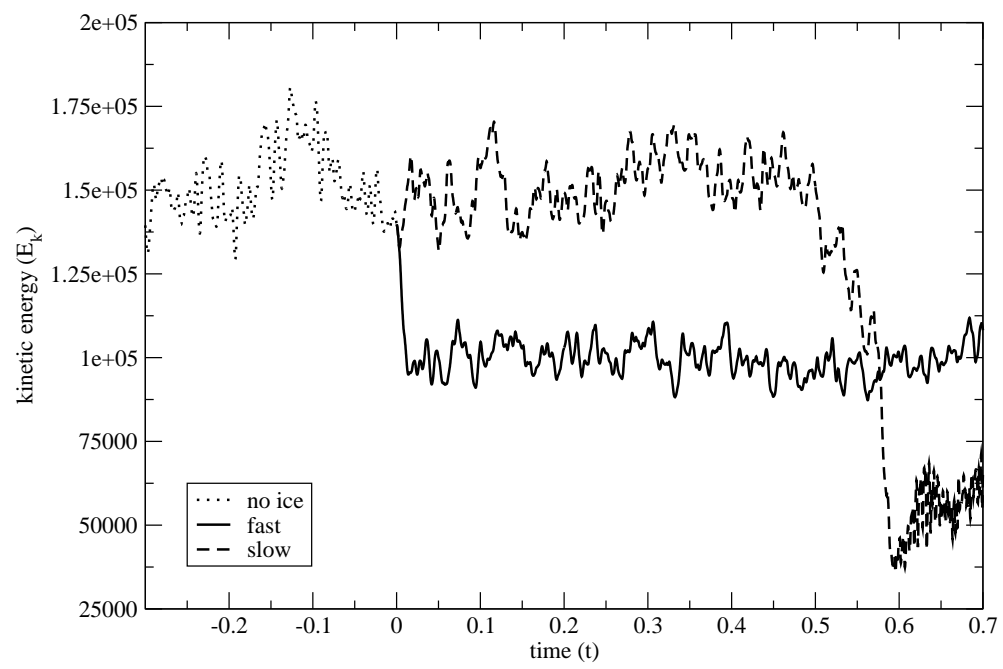

Figure 3: Evolution of kinetic energy for the two simulations of Fig. 2 Ice seeds are introduced at time $t=0$. Full line: $\gamma=10^{4}, w_{r}=150$ (fast growth). Broken line: $\gamma=10^{3}, w_{r}=10$ (slow growth). 
the large downwelling plumes. Fig. [5 shows a typical situation where we see small spots of ice next to the strongest downwelling plumes, and a large spot in a relatively quiescent region in the flow. The ice still forms mostly inside the coldest downward plumes, but now the process of ice formation is slow in comparison to the convection time scales and the ice forming has time to migrate towards quiescent regions. Looking again at Fig. 3 we remark that the total kinetic energy decreases as soon as a significant amount of ice is produced; this happens because most of the kinetic energy is concentrated in the most intense plumes: the ice that is produced in the strong downwelling plumes modifies the buoyancy force and tends to slow down the downward motion; as a consequence, the kinetic energy of those plumes is reduced.

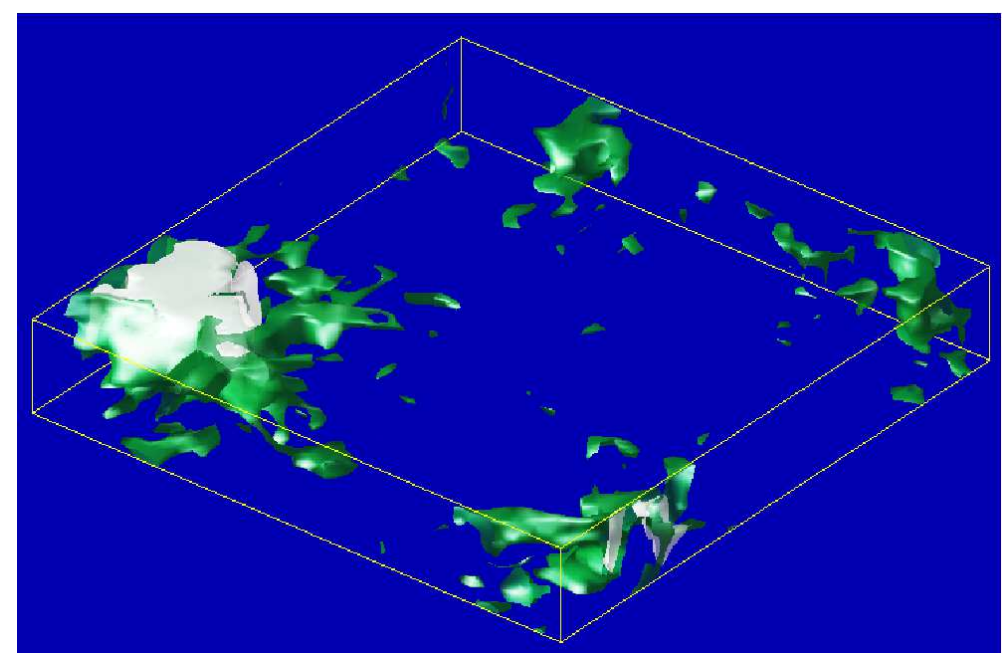

Figure 4: Isosurface of negative temperature fluctuations (-0.2, green) and isosurfaces of large ice concentration $\left(.1 C_{\max }\right.$, white). Snapshot taken at a temporal station during the exponential growth regime for a fast ice formation regime $\left(R a=10^{6}, \gamma=10^{4}, w_{r}=150\right)$.

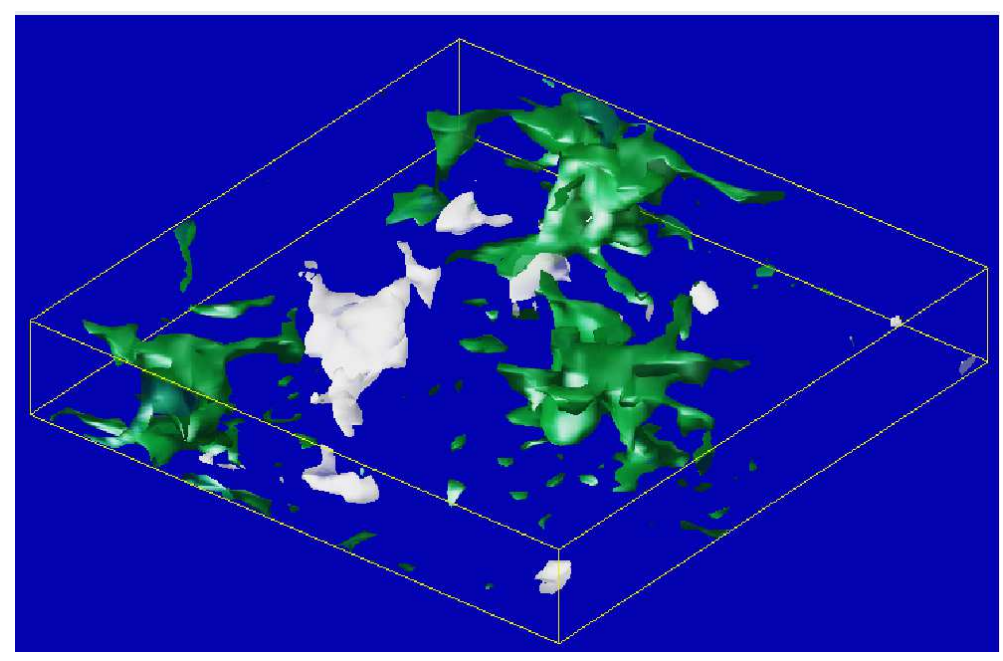

Figure 5: Isosurface of negative temperature fluctuations (-0.2, green) and isosurfaces of large ice concentration $\left(.1 C_{\max }\right.$, white $)$ for a slow ice formation regime $\left(R a=10^{6}, \gamma=10^{3}, w_{r}=10\right)$

In Figs. [6] and 7 we plot the growth rate for the linear phase as a function respectively of the rise velocity $w_{r}$ and of the thermal exchange coefficient $\gamma$. Each point in the figures is obtained 
by carrying on a full simulation until nonlinear saturation of ice concentration occurs. In the fast regime, the flow is almost frozen during the ice production and the growth is almost exponential; the growth rate is computed as the slope of ice concentration versus time in log scale (as in Fig. 2 (left)), without considering the initial transient and the final nonlinear regime. In the slow regime the value of the growth rate is determined as the slope of the line which fits at best the curve in the linear phase, omitting the initial transient.

We notice in Fig. 6 that the growth rate has a maximum at a particular value of the rise velocity which we denote $\bar{w}$. For $R a=10^{6}$ it is about $\bar{w} \simeq 75$.

Looking at the concentration profiles averaged in the horizontal directions (Fig. 8) we can distinguish three situations according to the value of $w_{r}$.

In the large rise velocity regime $\left(w_{r}>\bar{w}\right)$, the ice crystals are rapidly pushed upward and they cannot act as nuclei for ice formation; therefore the ice rate of growth decreases when rise velocity increases and most of the ice concentration is found near the top. If $w_{r} \sim \bar{w}$, the rise velocity is balanced by the downwelling velocity of the plumes in the upper layer of the domain, where the temperature is the lowest. Crystals remain confined in this region and their concentration grows rapidly. Finally if $w_{r}<\bar{w}$ the ice is transported down and exits the plumes where it was created. The ice spreads throughout the domain and the average concentration profile is almost flat (Fig. 8).

Note that the profiles observed by (21]) beneath lead in the Beaufort Sea (north of Alaska) and those obtained by (17]) from the numerical simulations of lead dynamics show a large presence of ice near the top of the layer; this is in agreement with the situation where $w_{r} \gtrsim \bar{w}$.

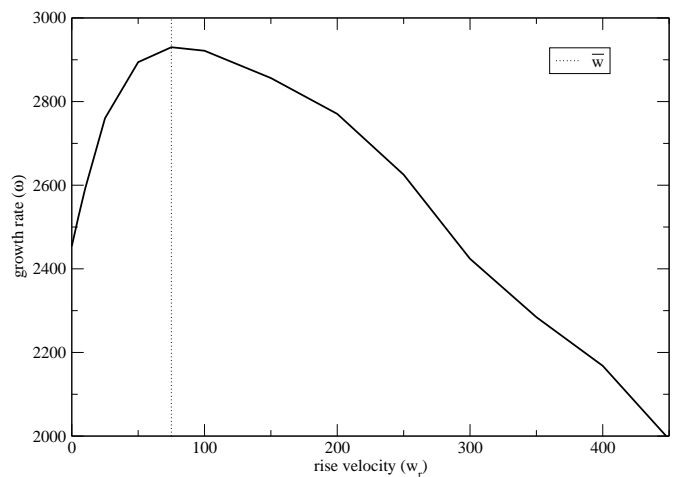

Figure 6: Growth rate of ice concentration versus rise velocity for $R a=10^{6}$ and $\gamma=$ $10^{4}$.

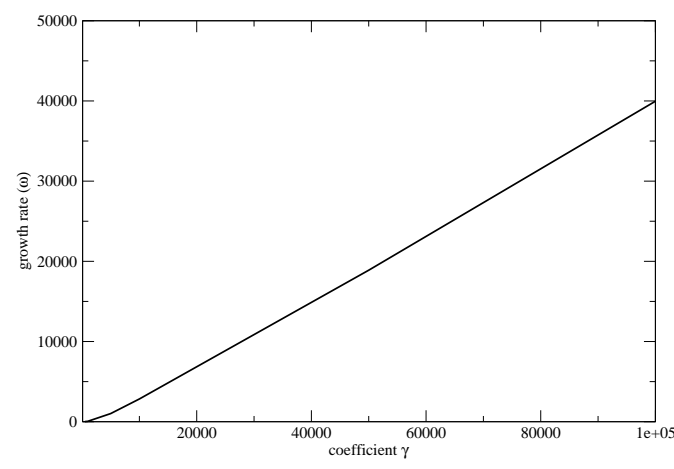

Figure 7: Growth rate of ice concentration versus $\gamma$ for $R a=10^{6}$ and $w_{r}=150$.

\subsection{A 1D model for fast ice formation}

We propose in this subsection a simple model that explains the behavior of the curves shown in Figs. 6 and 7 for the fast regime. We start from (20) and assume that inside the intense vertical plumes we can neglect horizontal velocities and horizontal derivatives. Therefore $C_{i}$ is a function of $z$ and $t$ and we get:

$$
\frac{\partial C_{i}}{\partial t}=-W \frac{\partial C_{i}}{\partial z}+\frac{1}{S} \frac{\partial^{2} C_{i}}{\partial z^{2}}+\Gamma C_{i}
$$

where:

$$
\frac{1}{S}=\frac{1}{S c}+\frac{1}{S c_{\mathrm{turb}}}, \quad W=w+w_{r}, \quad \Gamma=\gamma\left(T_{i}-T\right) .
$$

We perform an analysis into normal modes:

$$
C_{i}(t, z)=\widehat{C}(z) e^{\omega t}
$$




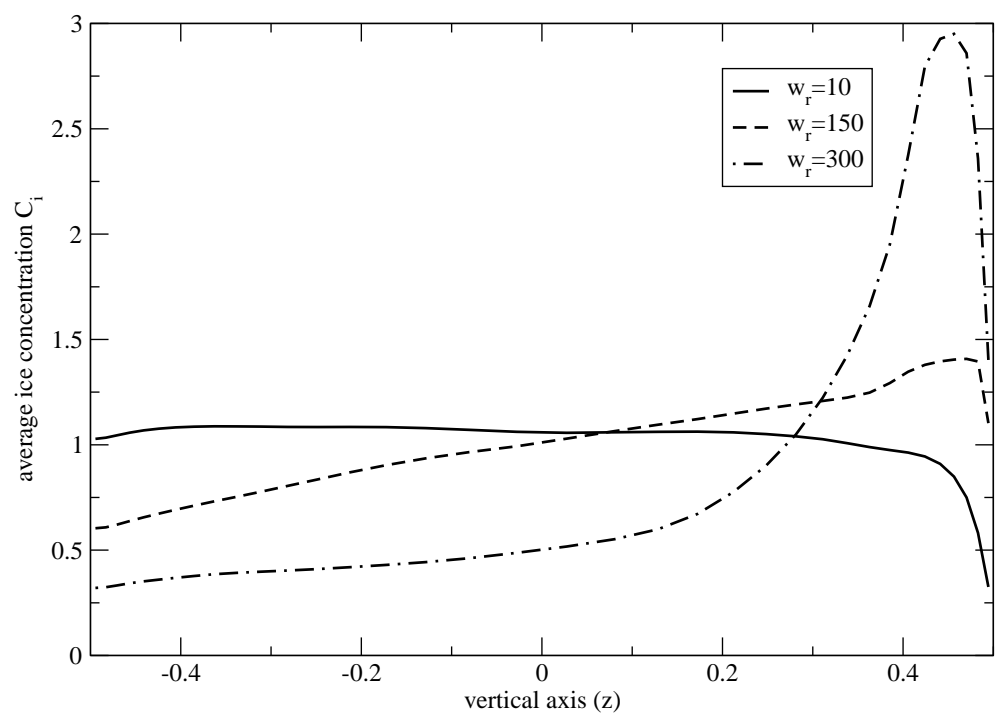

Figure 8: Average ice concentration for different values of $w_{r}\left(R a=10^{6}, \gamma=10^{3}\right)$

where $\omega \in \mathbb{C}$ is the eigenvalue and $\widehat{C}(z)$ the eigenvector:

$$
\widehat{C}^{\prime \prime}(z)-W S \widehat{C}^{\prime}(z)+(\Gamma-\omega) S \widehat{C}(z)=0
$$

$\left(\widehat{C}^{\prime}\right.$ and $\widehat{C}^{\prime \prime}$ denote derivatives with respect to $z$ ). Let us further assume that $W, \Gamma$ and $S$ are constant. We look for solutions with exponential dependence on $z$. Imposing the boundary conditions:

$$
\widehat{C}(-1 / 2)=\widehat{C}(1 / 2)=0,
$$

we get:

$$
\begin{gathered}
\omega=\Gamma-\frac{W^{2} S}{4}-\frac{n^{2} \pi^{2}}{S} \quad|n| \neq 0 \\
C_{i}(z, t)=a e^{\omega t} e^{\frac{W S z}{2}}\left(\left[1-(-1)^{n}\right] \cos n \pi z+i\left[1+(-1)^{n}\right] \sin n \pi z\right),
\end{gathered}
$$

where $n$ is a non vanishing integer and $a$ is an arbitrary constant. The case $n=0$ is degenerate; the growth rate is:

$$
\omega=\Gamma-\frac{W^{2} S}{4} .
$$

We can now impose only one boundary condition. If $W>0$ the eigenvector is:

$$
\widehat{C}(z)=a e^{\frac{W S z}{2}}(1-2 z)
$$

and the boundary condition is exact in $z=1 / 2$ and only approximately zero in $z=-1 / 2$. If $W<0$ :

$$
\widehat{C}(z)=a e^{\frac{W S z}{2}}(1+2 z)
$$

and the boundary condition is exact in $z=-1 / 2$ and only approximately zero in $z=1 / 2$.

The degenerate case is the one with the largest growth rate and it is therefore the most interesting. In Figs. 10 and 11] we plot the growth rate $\omega$ versus respectively $w_{r}$ and $\Gamma$ together with the actual values obtained by the LES simulations. The values of $W, \Gamma$ and $S$ are expressed by (22) and depend on typical values of vertical velocity $w$, temperature $T$ and turbulent Schmidt number $S c_{\text {turb }}$. Those values could be predicted by turbulent convection theories but we decide here to choose the values which fit at best the LES curve. As we see in Fig. 10] the fit is very 
good for rise velocities greater or equal to $\bar{w}$. The bad behaviour at smaller values is due to the fact that thei ice does not stay confined inside downwelling plumes and therefore the hypotheis of our model is not verified. In order to get a more quantitative agreement, we refine the model by dropping the assumption that $W$ and $\Gamma$ are constant. We compute the actual vertical profile of these functions by averaging the values obtained by the LES simulations inside the most intense downwelling plume (see Figs. 9).

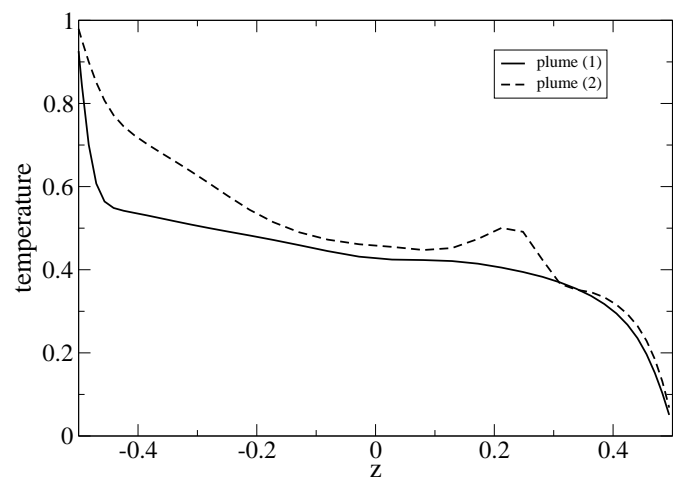

(a)

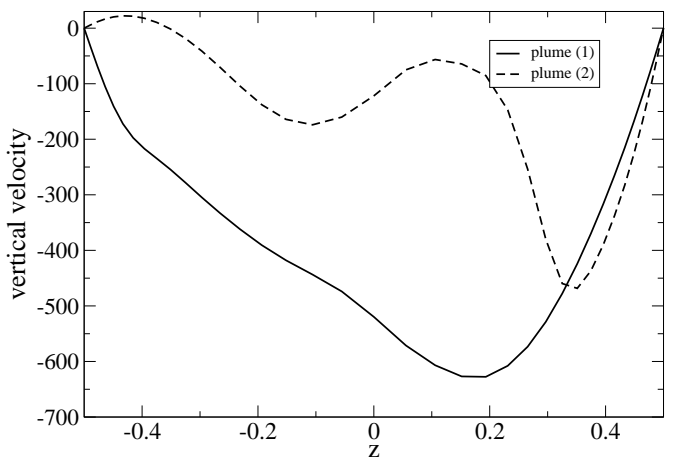

(b)

Figure 9: Vertical profile of temperature fluctuations (a) and vertical velocity (b) of the two most most intense downwelling plume. Data are averaged over the horizontal dimension of the plume. $R a=10^{6}, \gamma=10^{4}, w_{r}=150$.

The differential eigenvalue problem becomes:

$$
\left\{\begin{array}{l}
\frac{1}{S} \widehat{C}^{\prime \prime}-W(z) \widehat{C}^{\prime}+\Gamma(z) \widehat{C}=\omega \widehat{C}, \\
\widehat{C}(-1 / 2)=\widehat{C}(1 / 2)=0,
\end{array}\right.
$$

for prescribed functions $W(z)$ and $\Gamma(z)$. The solution is no more analytical and we compute it numerically. We adopt a spectral technique: we expand the solution in series of truncated Chebyshev polynomials and we discretize the problem over the Gauss-Lobatto grid points:

$$
z_{j}=\frac{1}{2} \cos \frac{\pi j}{N} \quad j=0, \ldots, N .
$$

We obtain a linear algebraic generalized eigenvalue problem:

$$
A \widehat{C}=\omega B \widehat{C},
$$

where $A$ and $B$ are $(N+1) \times(N+1)$ matrices. A method to solve such problem is to use the QZ algorithm. Such algorithm gives the full spectrum of eigenvalues/eigenvectors. Another method is to use iterative techniques (Krylov based methods), such as the Arnoldi method or the unsymmetric Lanczos method [2]. This method gives few eigenvalues corresponding to the least stable part of the spectrum. For our problem we have implemented both the QZ and the Arnoldi method and we have checked that the results produced by both techniques are always similar.

The growth rates obtained by solving (25) with the vertical profiles of the two most intense downwelling plumes and those given by the constant coefficient model (24) are shown in Figs. [10 and [1] From Fig. 10 we observe that when the rise velocity is far from the peak value $\bar{w}$, the growth rate predicted by our model underestimates the actual value (this occurs for about 
$w_{r}>300$ and $\left.w_{r}<75\right)$. The reason is that when the growth rate is small the flow cannot be considered steady during the exponential ice growth, but rather it fluctuates.

Figures 12 and 13 are similar to Figs. [10 and 11] except for the Rayleigh number that is $10^{8}$ instead of $10^{6}$. We see that the results obtained at $R a=10^{6}$ are still valid, thus they are robust and we can hope to extrapolate them to even larger Rayleigh numbers that are of interest for geophysical applications.

Some analytical progress far from the fast regime is possible provided $w_{r}$ is not too small (so to be able to balance the downwelling in the plumes). In this way, the permanence time $\tau_{p}$ of an ice crystal in a plume can be considered long and the growth process will be dominated by the plumes with the largest growth rate: $\left\langle\exp \left(\Gamma \tau_{p}\right)\right\rangle \sim \exp \left(\Gamma_{\mathrm{MAx}} \tau_{p}\right)$. Taking at the same time the limit $\Gamma \tau \ll 1$ with $\tau$ the typical plume circulation time, allows to treat the velocity and temperature fluctuations as a noise and to calculate averages in closed form.

Let us look at a single Lagrangian parcel, moving with velocity $w(z(t), t)+w_{r}$ (plus the effect of molecular diffusion) and endowed with an ice concentration $c(t)$ obeying:

$$
\frac{\mathrm{d} c(t)}{\mathrm{d} t}=\gamma\left[T_{i}-T(z(t), t)\right] c(t) .
$$

Identify with tilde and overbar, the fluctuating and mean field component of $w$ and $T$ at the parcel position $z: w(z, t)=\bar{w}(z, t)+\tilde{w}(t) ; T(z, t)=\bar{T}(z, t)+\tilde{T}(t)$ In the limit $\tau \rightarrow 0$, the equations for $z(t)$ and $c(t)$ can be written in the form:

$$
\left\{\begin{array}{l}
\mathrm{d} z=\left[w_{r}+\bar{w}(z, t)\right] \mathrm{d} t+\sigma_{\tilde{w}} \tau^{1 / 2} \mathrm{~d} B_{1}+(2 / S c)^{1 / 2} \mathrm{~d} B_{2} \\
\mathrm{~d} c=\gamma\left[T_{i}-\bar{T}(z, t)\right] c \mathrm{~d} t+\gamma \sigma_{\tilde{T}} \tau^{1 / 2} c \mathrm{~d}^{(S)} B_{3}
\end{array}\right.
$$

where the $\mathrm{d} B_{i}$ are Brownian increments, that, for simplicity, we take as independent: $\left\langle\mathrm{d} B_{i}\right\rangle=0$, $\left\langle\mathrm{d} B_{i} \mathrm{~d} B_{j}\right\rangle=\delta_{i j} \mathrm{~d} t$. The superscript in $\mathrm{d}^{(S)} B_{3}$ indicates Stratonovich prescription 4, and arises from the short correlation time limit $\int_{t}^{t+\tau} c\left(t^{\prime}\right) \tilde{T}\left(t^{\prime}\right) \mathrm{d} t^{\prime} \simeq \frac{1}{2} \sigma_{\tilde{T}} \tau^{1 / 2}[c(t)+c(t+\tau)]\left[B_{3}(t)-B_{3}(t+\tau)\right]$. This leads to a correction to the mean growth rate, and the second of eq. (6.2) will take the form, back to the standard Itô prescription:

$$
\mathrm{d} c=\gamma\left[T_{i}-\bar{T}(z, t)+\frac{1}{2} \gamma \sigma_{\tilde{T}}^{2} \tau\right] c \mathrm{~d} t+\gamma \sigma_{\tilde{T}} \tau^{1 / 2} c \mathrm{~d} B_{3} .
$$

The PDF $\rho(z, c ; t)$ will then obey the Fokker-Planck equation:

$$
\frac{\partial \rho}{\partial t}+\frac{\partial}{\partial z}\left[\left(w_{r}+\bar{w}\right) \rho\right]+\gamma \frac{\partial}{\partial c}\left[\left(T_{i}-\bar{T}+\frac{1}{2} \gamma \sigma_{\tilde{T}}^{2} \tau\right) c \rho\right]=\frac{\partial^{2}}{\partial z}(\rho / \bar{S})+\frac{1}{2} \frac{\partial^{2}}{\partial c}\left(\gamma \sigma_{\tilde{T}}^{2} \tau c^{2} \rho\right),
$$

where $\bar{S}^{-1}=S c_{0}^{-1}+\sigma_{\tilde{w}}^{2} \tau / 2$ may include non-subgrid contributions to the fluctuations. The concentration field resulting from this averaging procedure will be: $\bar{C}_{i}(z, t)=\int \rho(c, z ; t) c \mathrm{~d} c$, and, substituting into eq. (26), we obtain:

$$
\frac{\partial \bar{C}_{i}}{\partial t}+\frac{\partial}{\partial z}\left[\left(w_{r}+\bar{w}\right) \bar{C}_{i}\right]=\frac{\partial^{2}}{\partial z} \bar{C}_{i} / \bar{S}+\gamma\left(T_{i}-\bar{T}+\frac{1}{2} \gamma \sigma_{\tilde{T}}^{2} \tau\right) \bar{C}_{i} .
$$

Thus, the temperature fluctuations lead to a net increase in the ice concentration growth rate:

$$
\Gamma \rightarrow \Gamma+\frac{1}{2} \gamma \sigma_{\tilde{T}}^{2} \tau
$$

Neglecting this effect, as shown in Fig. 10 leads to underestimating the actual growth rate. Similarly, in Fig. [1] the slope of theoretical curves should be increased, which is precisely what eq. (28) does.

Notice that Eq. (21) would be recovered from (27) (as it should) if no fluctuating component were singled out of the $w$ and $T$ fields. 


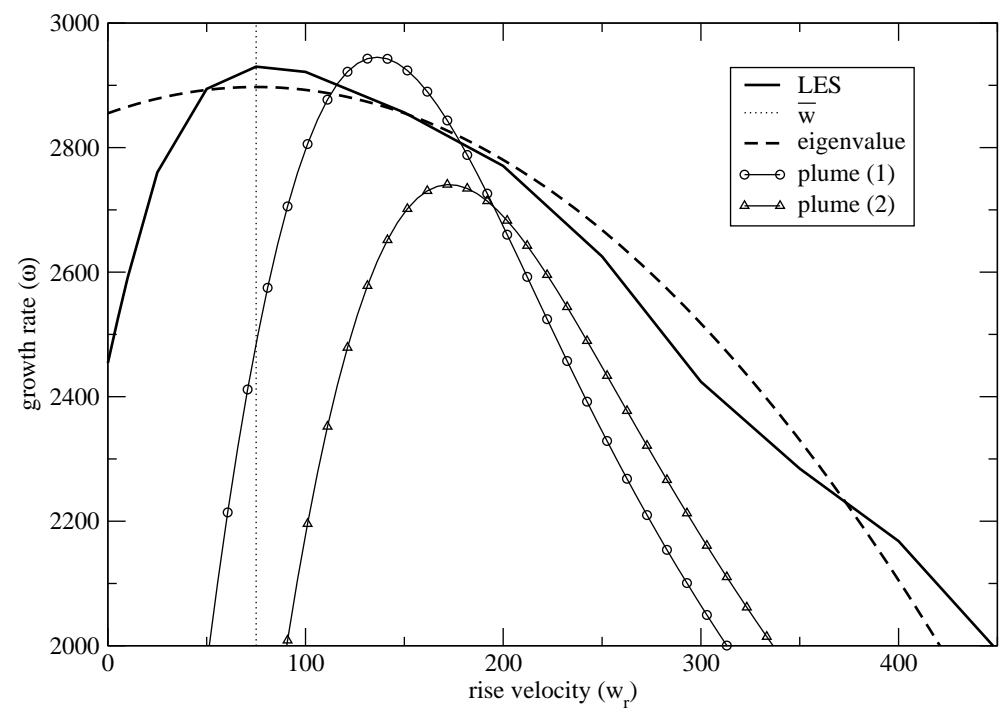

Figure 10: Growth rate of ice concentration versus rise velocity for $\gamma=10^{4}$ and $R a=10^{6}$. Full line: Growth rate from LES simulations.

Broken line: eigenvalue given by (24).

Line with circles: eigenvalue of problem (25) for the most intense downwelling plume.

Line with triangles: eigenvalue of problem (25) for the second most intense downwelling plume

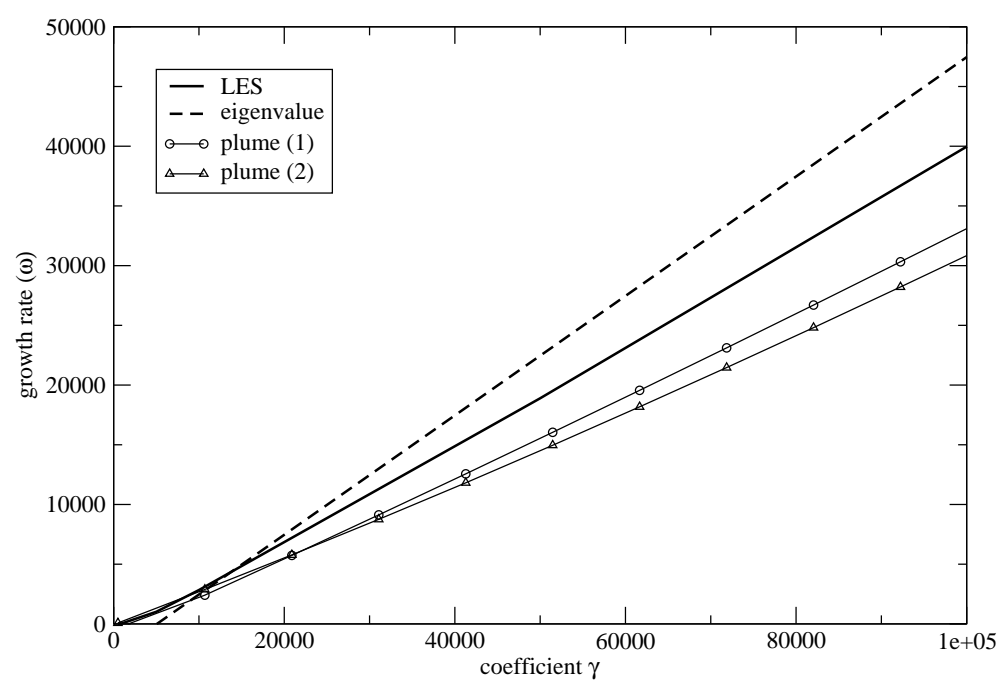

Figure 11: Growth rate of ice concentration versus $\gamma$ for $w_{r}=150$ and $R a=10^{6}$. For the legend see Fig [10] 


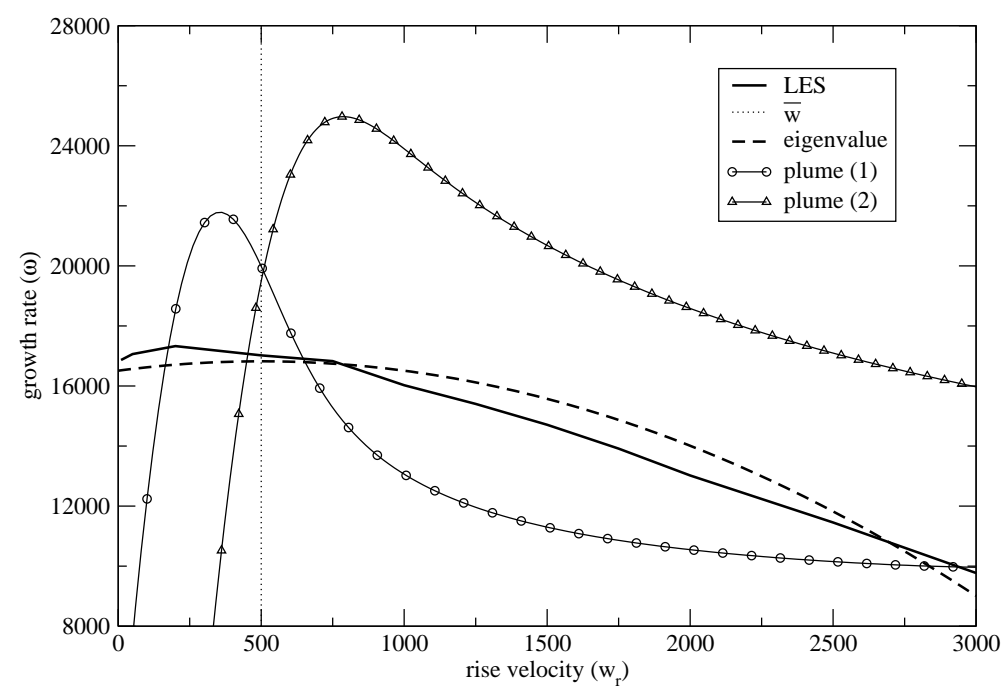

Figure 12: Growth rate of ice concentration versus rise velocity for $\gamma=10^{5}$ and $R a=10^{8}$

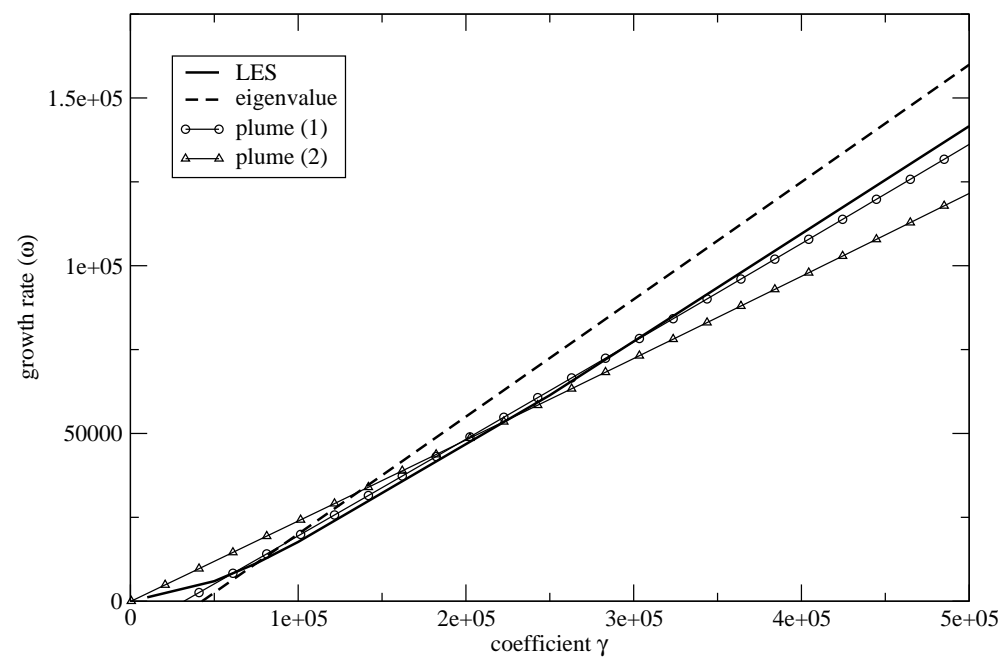

Figure 13: Growth rate of ice concentration versus $\gamma$ for $w_{r}=1000$ and $R a=10^{8}$ 


\subsection{Non linear phase}

The nonlinear saturation occurs when the ice concentration (rescaled by the factor $\alpha$, see (20) reaches values of the order of unity: the density of the mixture varies by amounts of order $\alpha$ and the buoyancy force in (18) is significantly affected. One must bear in mind that the Boussinesq approximation breaks down when ice concentration exceeds values of order unity; therefore results of the nonlinear stage must be taken with caution: they give qualitative indications but they cannot be used to assert quantitative conclusions.

In Fig. 14 we plot the temporal evolution of total ice concentration for three simulations with different values of $\beta$. The linear stage is insensitive to the value of $\beta$ because the ice concentration is too low to produce any change in temperature fluctuations. However we see that the nonlinear saturation is affected by the value of $\beta$ and the general trend is that for larger values of $\beta$ the ice saturation levels tend to decrease.

In Fig. 15] we see that the presence of ice inhibits convection.

In Fig. [16 we plot the average vertical temperature profiles during the nonlinear stage. We see that temperature tends to increase when $\beta$ decreases. Lower values of $\beta$ correspond to larger values of ice concentration, as we saw in Fig. 14 Therefore when total ice concentration increases the temperature of the flow tends to increase. This can be explained by the fact that during the phase transition in the freezing process there is an emission of heat. Therefore more ice production corresponds to more heat in the flow and the average temperature tends to increase.

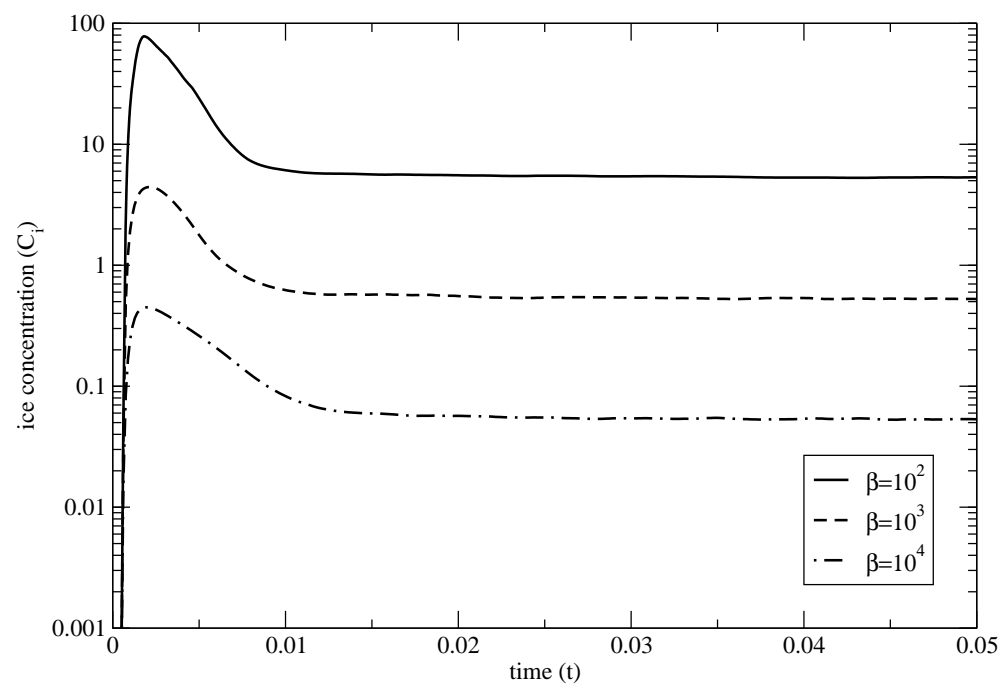

Figure 14: Evolution of ice concentration for different values of $\beta$ for $R a=10^{6}, w_{r}=100$ and $\gamma=10^{5}$

\subsection{Free surface flow}

In subsection 6.1 we were interested in the ice produced in the regions far from the upper layer, and therefore we adopted the simple boundary condition $C_{i}=0$ for the ice concentration at the surface.

In this subsection we want to study if and how the upper boundary conditions affect the ice concentration profile and growth. There is still no general consensus on how to obtain no upward ice flux at the air-water interface. Some authors (15], 14] and 19]) impose Neumann condition $\partial C_{i} / \partial z=h_{c}$, with $h_{c} \neq 0$, in order to represent the seeding effect due to the mass exchange with the atmosphere. It is still not clear, however, how to model the flux $h_{c}$. Other authors [17. propose to account for the ice crystals escaping across the air-water interface by accumulating 


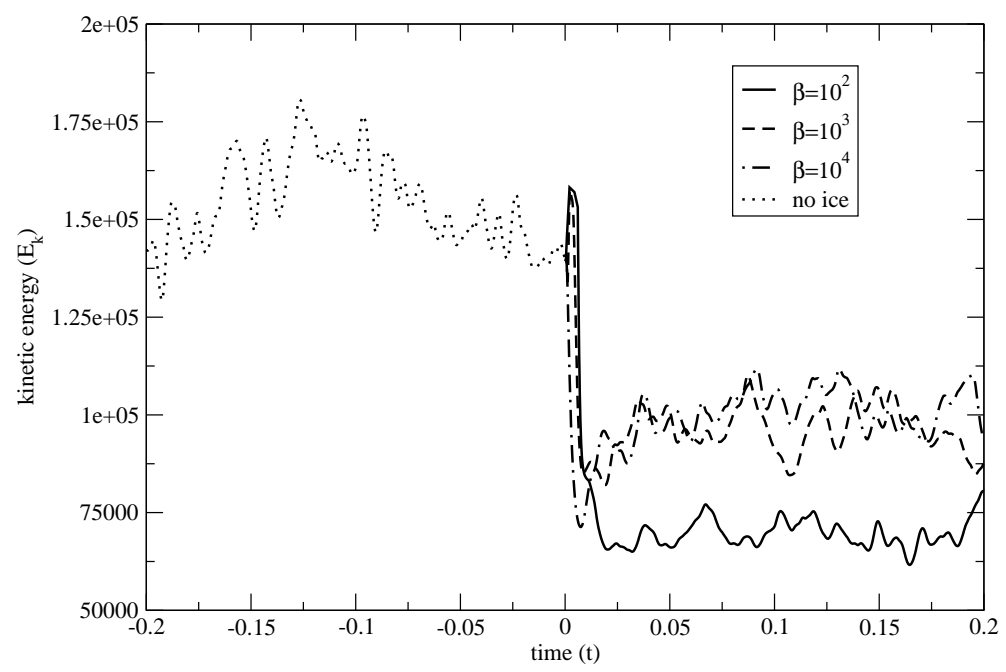

Figure 15: Evolution of kinetic energy for different values of $\beta$ for $R a=10^{6}, w_{r}=100$ and $\gamma=10^{5}$

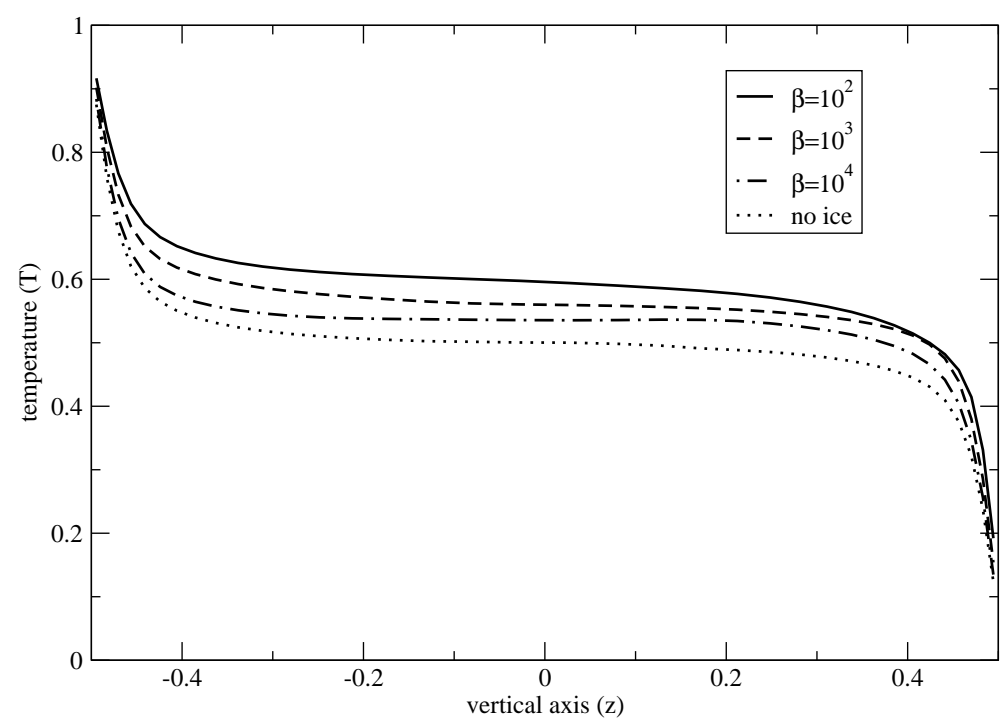

Figure 16: Mean vertical temperature for different values of $\beta$ for $R a=10^{6}, w_{r}=100$ and $\gamma=10^{5}$ 
them in a floating ice slab. Here we consider the situation where no ice sheet has formed at the water surface, therefore the water surface is a free surface, and we neglect the seeding effect. The suitable boundary conditions for this situation are free slip conditions for velocity and total vanishing flux for ice concentration:

$$
w=0, \quad \frac{\partial u}{\partial z}=\frac{\partial v}{\partial z}=0, \quad \frac{\partial C_{i}}{\partial z}-w_{r} S C_{i}=0
$$

where $S$ is the effective Schmidt number: $\frac{1}{S}=\frac{1}{S c}+\frac{1}{S c_{\text {turb }}}$. The only difference with subsection 6.1 is that we impose vanishing total ice concentration flux instead of vanishing convective flux.

In Fig. [18 we plot the vertical profile of ice concentration averaged over horizontal directions; we also plot the one obtained using the boundary conditions of subsection 6.1 We see that the two curves differ significantly near the surface, where the new boundary conditions produce a peak of ice concentration. Far from the upper boundary however the two profiles look very similar. In Fig. 17 we present a snapshot taken during the linear phase of the large downwelling plumes and of the ice concentration. Comparison with Fig. 4 confirms that the main difference is the accumulation of ice near the water surface.

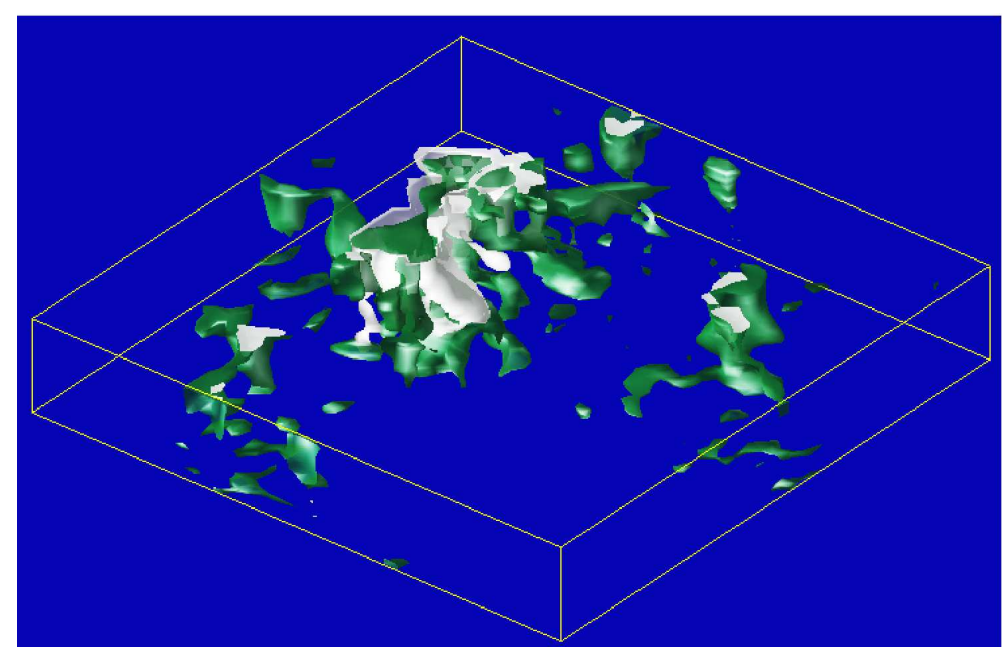

Figure 17: Isosurface of negative temperature fluctuations $(-0.2$, green) and isosurfaces of large ice concentration $\left(.1 C_{\max }\right.$, white). Snapshot taken at a temporal station during the exponential growth regime for a fast ice formation regime $\left(R a=10^{6}, \gamma=10^{4}, w_{r}=150\right)$.

We compare next the growth rate of ice concentration for the new and old boundary conditions: in Fig. [19] we present the growth rate of ice concentration as a function of the rise velocity $w_{r}$. We observe that the two curves are similar. When the rise velocity $w_{r}$ is small, we observe that the growth rate using the new boundary conditions is larger. This can be explained by the fact that these boundary conditions allow production of ice near the surface.

We can thus conclude that the new boundary conditions only affect the ice produced at the top of the layer, but they do not affect significantly the overall ice production rate and the ice concentration profile in the deep layers.

\section{Conclusions}

In this paper we have explored the sensitivity of the ice formation process to a set of nondimensional parameters: the ice melting/freezing source term $\gamma$, the ratio between the latter and the temperature source term $\beta$, and the ice particle rise velocity $\widehat{w}_{r}$. Regarding the Rayleigh number $R a$, we have considered moderately turbulent regimes up to $R a=10^{8}$. 


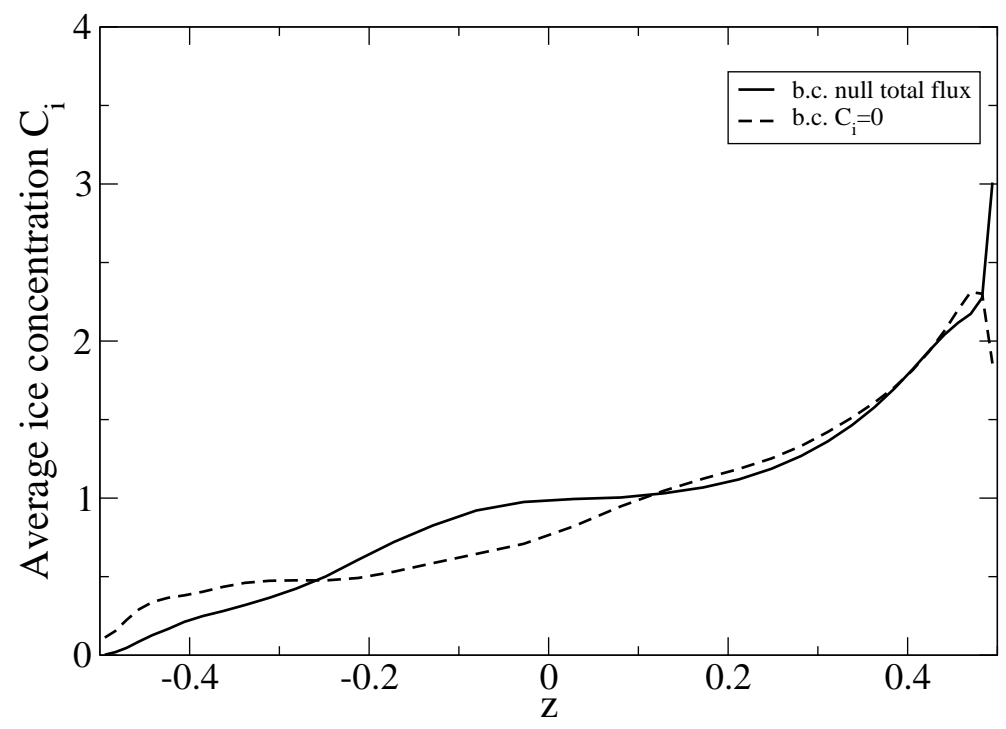

Figure 18: Vertical profile of ice concentration averaged over the horizontal directions at a temporal frame during the exponential growth regime. The two curves differ by the boundary conditions adopted at the water-air interface. The parameters are $R a=10^{6}, \gamma=10^{4}, w_{r}=150$.

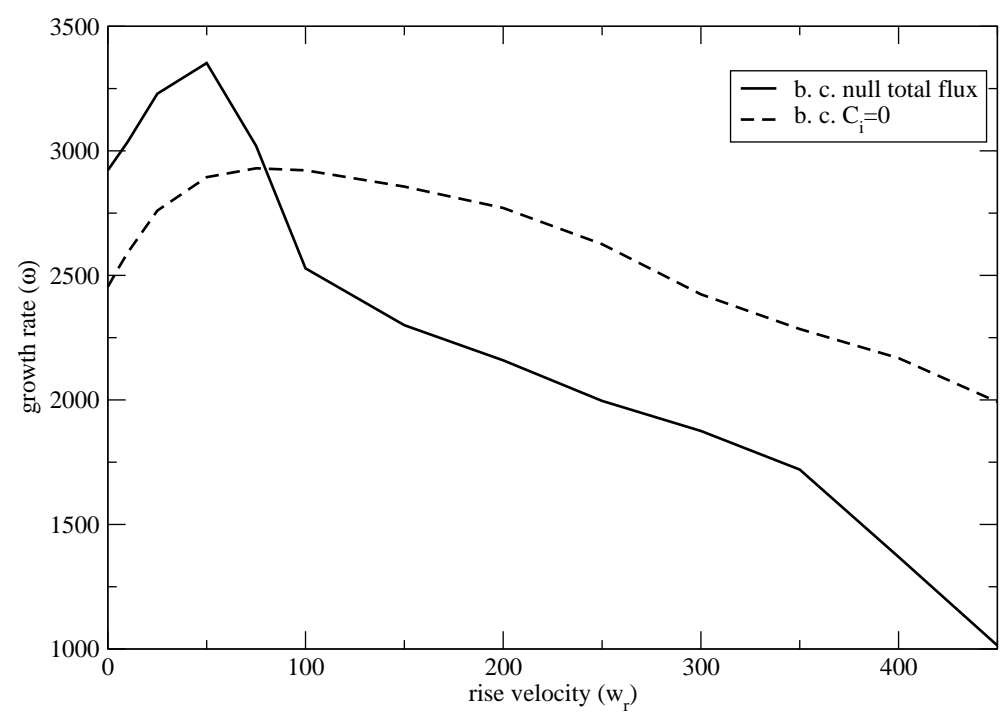

Figure 19: Growth rate of ice concentration versus rise velocity for different boundary conditions $\left(R a=10^{6}, \gamma=10^{4}\right)$ 
We have modeled the mixture of ice crystals and water as a two-phase medium composed of water and ice particles of fixed diameter. The equations describing the system are the classical balance equations for mass, momentum and energy in the ice-water mixture, supplemented by an advection-diffusion equation for the ice mass concentration with an additional source term, which account for the net ice production during melting or freezing process. In order to account for the net heat exchange during the same process, an additional source term has been introduced in the temperature equation. The simulation of the complete equations, however, is subject to numerical instability due to large round-off errors. The origin of such error is the factor $R a / \bar{\alpha}$ ( $\bar{\alpha}$ is the coefficient of thermal expansion) in the buoyancy term, which, for values of $R a$ of interest $\left(R a \geq 10^{8}\right)$, is larger than $10^{14}$. Therefore, we have developed and applied the Boussinesq approximation and we have integrated numerically the set of equations making use of a numerical code based on second order finite difference. A dynamic LES model has been used in order to cope with the subgrid scales that are not considered in the simulations.

The sensitivity analysis has shown that the ice particle rise velocity and the ice concentration source term coefficient $\gamma$ significantly affect the frazil ice dynamics. In the large rise velocity regime, the ice crystals are rapidly pushed upward and they cannot act as nuclei for ice formation; in the opposite regime of small rise velocity they tend to be transported downwards in warmer regions where they tend to melt down. The maximum of ice production is obtained in those situations where the rise velocity is of the same order of magnitude of the peak rise velocity $\bar{w}$. We have developed a simple model which captures the trend of the growth rate as a function of the relevant parameters. The model has been derived in the approximation where the ice is produced far beneath the water surface, however it is found to be valid also for free surface flow.

The parameter which plays a key role in fixing the concentration value at the statistically steady state is the heat exchange source term coefficient $\beta$. The greater is $\beta$, the lower is the domain averaged ice concentration. Moreover, the presence of more frazil ice crystals induces a rapid fall in the kinetic energy and this energy lowering increases as the saturated ice concentration increases.

The Boussinesq approximation holds only when frazil ice mass concentration is very small, i.e. of the same order of the small non-dimensional parameter $\bar{\alpha}=\alpha \Delta T$. So, the Boussinesq approximation represents well the initial stage of ice formation, but it breaks down when the ice concentration becomes significant. Moreover, at significant ice concentrations, the particles interaction and the effective viscosity increment due to the presence of the ice particles should be taken into account $[3]$.

Other effects that were not included in the present study and which we postpone for the future are: the inclusion of salinity, the occurrence of different classes of ice crystals with different size, the effect of different conditions at the top of the layer, for example a horizontal wind blowing over the water, or the presence of an ice shelf.

\section{Acknowledgments}

The work of L.P. was funded by the EU Project 'Convection' and, partially, by PNRA, the Italian Program for Antarctic Res. The authors would like to thank Dr. F. Parmiggiani and Prof. P. Wadhams for valuable comments and stimulating discussions.

\section{References}

[1] C.E. Adams and G.L. Weatherly. Some effects of suspended sediment stratification on an oceanic bottom boundary layer. J. Geophys. Res., 86:4161-4172, 1981.

[2] F. Chatelin. Eigenvalues of matrices. Wiley, Chichester, 1993. Enlarged Translation of the French Edition with Masson. 
[3] P. Olla G. De Carolis and L. Pignagnoli. Effective viscosity of grease ice in linearized gravity waves. J. Fluid Mech., 86, 2005.

[4] C. W. Gardiner. Handbook of stochastic methods. Springer-Verlag, 1985.

[5] Gosnik and T.E. Osterkamp. Measurements and analyses of velocity profiles and frazil icecrystal rise velocities during periods of frazil ice formation in rivers. Annals of Glaciology, 4:79-83, 1983.

[6] P.R. Holland and D.L. Feltham. Frazil dynamics and precipitation in a water column with depth-dependent supercooling. J. Fluid Mech., 530:101-124, 2005.

[7] J.P. Holman. Heat transfer. McGrow-Hill, 1977.

[8] J.N. Hunt. On the turbulent transport of a heterogeneous sediment. Q. J. Mech. Appl. Math., $22: 235-246,1969$.

[9] A. Jenkins and A. Bombosch. Modeling the effects of frazil ice crystals on the dynamics and thermodynamics of ice shelf water plumes. J. Geophysical Res., 100 - C4:6967-6981, 1995.

[10] H.R. Kivisild. River and lake ice terminology. Int. Assoc. Hydraul. Res., paper 1.0, 14 pp, 1970.

[11] L.D. Landau and E.M. Lifshitz. Fluid Mechanics. Pergamon, 1959.

[12] H. Le and P. Moin. An improvement of fractional step methods for the incompressible NavierStokes equations. J. Comp. Phys., 92:369-379, 1991.

[13] S. Martin. Frazil ice in rivers and oceans. Ann. Rev. Fluid Mech., 13:379-397, 1981.

[14] A. Omstedt. On supercooling and ice formation in turbulent sea-water. J. Glaciol., 31(109):263-271, 1985.

[15] A. Omstedt and U. Svensson. Modelling supercooling and ice formation in a turbulent ekman layer. J. Geophysical Res., 89:735-744, 1984.

[16] W. Press, B. Flannery, S. Teukolsky, and W. Vetterling. Numerical Recipes in C. Cambridge University Press, 1990.

[17] E.D. Skyllingstad and D.W. Denbo. Turbulence beneath sea ice and leads: a coupled sea ice / large eddy simulation study. J. Geophysical Res., 106:2477-2497, 2001.

[18] L.H. Smedsrud and A.D. Jenkins. Frazil ice formation in an ice shelf water plume. J. Geophysical Res., 109:C03025, 2004.

[19] U. Svensson and A. Omstedt. Numerical simulation of frazil ice dynamics in the upper layer of the ocean. Cold Regions Science and Technology, 28:29-44, 1998.

[20] V.C. Wong. A proposed statistical-dynamic closure method for the linear or nonlinear subgridscale stresses. Phys.Fluids A, 4(5)(5):1080-1082, May 1992.

[21] C.A. Paulson W.S. Pegau and J.R.V. Zaneveld. Optical measurements of frazil concentration. Cold Regions Science and Technology, 24:341-353, 1985. 